\title{
EXISTENCE AND ASYMPTOTIC \\ BEHAVIOUR FOR A DEGENERATE \\ KIRCHHOFF-CARRIER MODEL WITH \\ VISCOSITY AND NONLINEAR BOUNDARY \\ CONDITIONS
}

\section{M.M. CAVALCANTI, V.N. DOMINGOS CAVALCANTI, J.A. SORIANO and J.S. PRATES FILHO}

\footnotetext{
Abstract

The present paper studies the existence and uniqueness of global solutions and decay rates to the nonlinear hyperbolic problem

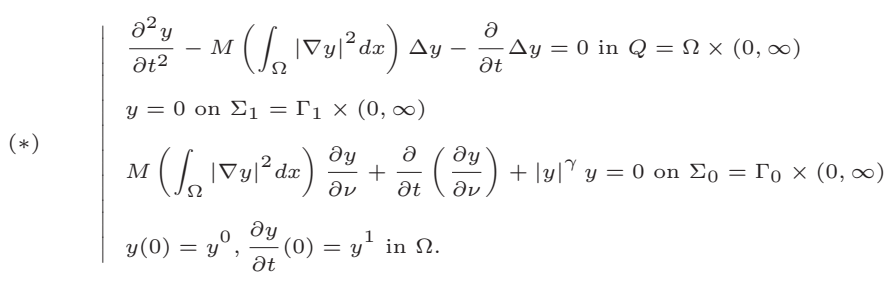

where $M$ is a $C^{1}$ function; $M(\lambda) \geq 0 ; \forall \lambda \geq 0$.
}

\section{Introduction}

Let $\Omega$ be a bounded domain of $\mathbf{R}^{n}$ and let $\Gamma$ denote its $C^{2}$ boundary. Assume that $\Gamma$ consists of two parts, $\Gamma_{0}$ and $\Gamma_{1}$, with positive measure and such that $\Gamma_{0}$ and $\Gamma_{1}$ are closed and disjoint. Let $\nu$ be the unit normal vector pointing towards the exterior of $\Omega$ and let $\frac{\partial}{\partial \nu}$ denote the normal derivative.

Let $M \in C^{1}([0, \infty), \mathbf{R})$ be a function which verifies

$$
M(\lambda) \geq 0 ; \quad \forall \lambda \geq 0
$$

2000 Mathematics Subject Classification: 35B40, 35L80.

Servicio de Publicaciones. Universidad Complutense. Madrid, 2001 
The aim of this paper is to prove existence and uniqueness of strong and weak solutions and to show that these solutions decay to zero uniformly when $t$ goes to infinity.

The motivation for this problem comes from the small vibrations of an elastic string whose mathematical model, c.f. J. L. Lions [12], is given by the following Kirchhoff-Carrier equation

$$
\rho h \frac{\partial^{2} y}{\partial t^{2}}=\left(P_{0}+\frac{E h}{2 L} \int_{0}^{L}\left|\frac{\partial y}{\partial x}(x, t)\right|^{2} d x\right) \frac{\partial^{2} y}{\partial x^{2}} ; \quad 0 \leq x \leq L, t \geq 0 .
$$

Here $y$ is the lateral displacement at the space coordinate $x$ and the time $t, \rho$ the mass density, $h$ the cross-section area, $L$ the length, $E$ the Young's modulus and $P_{0}$ the initial axial tension.

When $n=1$, for instance, problem $\left(^{*}\right)$ describes the nonlinear vibrations of an one dimensional string which is clamped in one end and is free in the other end in the sense that it can move up and down subject to nonlinear effects caused by the function $f(y)=|y|^{\gamma} y$.

Hypothesis $M(0)=0$ and $M(\lambda)>0$ in a neighbourhood of the origin (see assumption (2.5) below)raised from the fact that we are interested in the case $P_{0}=0$.

A lot of papers have been devoted to this context. In this direction we can cite, for instance, Menzala [17], Arosio-Spagnolo [1], Rivera [21] and Ebihara-Milla Miranda-Medeiros [5]. Let us also mention the results of Yamada [24, 25], Vasconcellos-Teixeira [23], Muñoz Rivera [18] and Medeiros-Milla Miranda [14] which are in connection with damped problems. It is interesting to observe that problems without viscosity, that is, when $\Delta y^{\prime}=0$ and assuming that $M=1$ and a feedback occurs on the boundary were studied by many authors, see Quinn and Russel [20], Chen [4], Lagnese [8,9], Komornik and Zuazua [7], Lasiecka and Tataru [10] and Cavalcanti, Domingos Cavalcanti and Soriano [2].

In spite of the importance of the subject, there are relatively few mathematical results in the presence of nonlinear boundary conditions combined with the nonlinearity due to the function $M$. To deal with this kind of problem is the contribution of this paper. Comparing the present paper with the problems considered in [11] and [16], where the term $\Delta y_{t}$ was not added, we observe that in both cases the function $M$ is not supposed to degenerate which contrasts with assumption (1.1). 
In order to obtain the existence of solutions in [3], the authors employ Galerkin's method and, as they are not allowed to use basis formed by eigenfunctions, make use of arguments which are only valid when the only nonlinearity is given by $M=M(t)$. However, in the present paper, we have to deal with a nonlinear boundary condition and the degeneracy of the function $M$, which do not permit us to repeat the same arguments used in [3]. To overcome these difficulties is the goal of our work.

To obtain the uniform decay rates of the energy (c.f. Theorem 2.1 below)

$$
\begin{aligned}
E(t)= & \frac{1}{2}\left[\int_{\Omega}\left|y^{\prime}(x, t)\right|^{2} d x+\hat{M}\left(\int_{\Omega}|\nabla y(x, t)|^{2} d x\right)\right]+ \\
& +\frac{1}{\gamma+2} \int_{\Gamma_{0}}|y(x, t)|^{\gamma+2} d \Gamma
\end{aligned}
$$

where

$$
\hat{M}(\lambda)=\int_{0}^{\lambda} M(s) d s
$$

we use the perturbed energy method, see Zuazua [7,26], combined with techniques from Muñoz Rivera [18].

Our paper is organized as follows. In section 2 we give the notations and state the main result. In section 3 we study existence and uniqueness of strong and weak solutions while in section 4 we obtain the uniform decay rates for solutions obtained in section 3 .

\section{Notations and main results}

In this section we present some notations which will be used throughout this paper and will state the main result.

Let

$$
V=\left\{v \in H^{1}(\Omega) ; \quad v=0 \quad \text { on } \Gamma_{1}\right\}
$$

which indowed with the topology given by the norm $|\nabla \cdot|_{L^{2}(\Omega)}$ is a Hilbert subspace of $H^{1}(\Omega)$.

We denote

$$
\begin{gathered}
(u, v)=\int_{\Omega} u(x) v(x) d x ; \quad(u, v)_{\Gamma_{0}}=\int_{\Gamma_{0}} u(x) v(x) d x \\
|u|^{2}=\int_{\Omega}|u(x)|^{2} d x,|u|_{\Gamma_{0}}^{2}=\int_{\Gamma_{0}}|u(x)|^{2} d \Gamma,\left.|| u\right|_{p, \Gamma_{0}} ^{p}=\int_{\Gamma_{0}}|u(x)|^{p} d \Gamma
\end{gathered}
$$


and assume that

$$
0<\gamma \leq \frac{1}{n-2} ; n \geq 3 \text { or } \gamma>0 \text { if } n=1,2 .
$$

Now, we are in position to state our main results.

Theorem 2.1. Assume that (1.1) holds and

$$
\left\{y^{0}, y^{1}\right\} \in V^{2} .
$$

Then, the problem $(*)$ possesses a unique strong solution satisfying

$$
y \in L^{\infty}(0, \infty ; V), \quad y^{\prime} \in L^{\infty}(0, \infty ; V) ; \quad y^{\prime \prime} \in L^{2}\left(0, \infty ; L^{2}(\Omega)\right) .
$$

Besides, the following energy decay holds

$$
E(t) \leq C(1+t)^{-1}, \quad \forall t \geq 0,
$$

where $C$ is a positive constant.

Assuming that $M$ is non decreasing and, for some $\alpha>0$, satisfies the assumptions

$$
\liminf _{s \rightarrow 0^{+}} \frac{M(s)}{s^{\alpha}}>0 \quad \text { and } \quad M(s)=O\left(s^{\alpha}\right), s \rightarrow 0^{+},
$$

there exist positive constants $C$ and $N$ such that

$$
E(t)+\frac{1}{2}|\nabla y(t)|^{2} \leq C(1+t)^{-\frac{1}{\alpha}}, \quad \forall t \geq N
$$

and

$$
M\left(|\nabla y(t)|^{2}\right) \leq C(1+t)^{-1}, \quad t \geq N .
$$

Moreover, under the hypothesis

$$
M(\lambda) \geq \lambda_{0}>0 \text { for all } \lambda>0
$$

we obtain the exponential decay, that is, there exist positive constants $C, \theta$ such that

$$
E(t) \leq C \exp (-\theta t), \quad t \geq 0 .
$$

Remark. The assumption (2.5) means that the function $M(s)>0$ and it is bounded by a polinomial $P(s)=k s^{\alpha}(k>0)$ in an neighbourhood of 
the origin. Consequently $M(0)=0$ and the algebraic decay rates occur according to the exponent $\alpha$ of the polinomial $P(s)$ which dominates the function $M(s)$.

Theorem 2.2. Let

$$
\left\{y^{0}, y^{1}\right\} \in V \times L^{2}(\Omega)
$$

and assume that $M \in C^{1}(0, \infty)$. Then, problem $(*)$ possesses a unique weak solution in the class

$$
y \in C^{0}([0, \infty) ; V) ; \quad y^{\prime} \in C^{0}\left([0, \infty) ; L^{2}(\Omega)\right)
$$

and verify the decay rate given in (2.4).

Moreover, if $M$ is non decreasing and satisfies the assumptions given in (2.5), we have the decay rates obtained in (2.6) and (2.7) in the degenerate case and the decay given by (2.9) in the nondegenerate one.

\section{Existence and uniqueness of solutions}

Let us consider initially

$$
\left\{y^{0}, y^{1}\right\} \in V^{2} .
$$

The variational formulation associated with problem $(*)$ is given by

$$
\begin{gathered}
\left(y^{\prime \prime}(t), w\right)+M\left(|\nabla y(t)|^{2}\right)(\nabla y(t), \nabla w)+\left(\nabla y^{\prime}(t), \nabla w\right) \\
+\left(|y(t)|^{\gamma} y(t), w\right)_{\Gamma_{0}}=0 ; \quad \text { for all } w \in V .
\end{gathered}
$$

We represent by $\left(\omega_{j}\right)_{j \in \mathbf{N}}$ a basis in $V$, which is ortonormal in $L^{2}(\Omega)$, and by $V_{m}$ the subspace of $V$ generate by the m-first vectors $\omega_{1}, \cdots, \omega_{m}$. Let us define

$$
y_{m}(t)=\sum_{i=1}^{m} g_{i m}(t) \omega_{i},
$$

where $y_{m}(t)$ is the solution of the following Cauchy problem

$$
\begin{gathered}
\left(y_{m}^{\prime \prime}(t), \omega_{j}\right)+M\left(\left|\nabla y_{m}(t)\right|^{2}\right)\left(\nabla y_{m}(t), \nabla \omega_{j}\right)+\left(\nabla y_{m}^{\prime}(t), \nabla \omega_{j}\right) \\
+\left(\left|y_{m}(t)\right|^{\gamma} y_{m}(t), \omega_{j}\right)_{\Gamma_{0}}=0, \\
y_{m}(0)=y_{0 m} \rightarrow y^{0} \quad \text { in } V \quad \text { and } \quad y_{m}^{\prime}(0)=y_{1 m} \rightarrow y^{1} \quad \text { in } V .
\end{gathered}
$$


The above approximate system is a normal one of ordinary differential equations and has a solution in $\left[0, t_{m}\right)$. The extension of the solution to the whole interval $[0, \mathrm{~T}]$ is a consequence of the first estimate which we are going to obtain below.

\subsection{A priori estimates}

\section{The first estimate.}

Multiplying (3.4) by $g_{j m}^{\prime}(t)$ and summing over $j$ from (1.4) we have

$$
\begin{aligned}
& \frac{d}{d t}\left\{\frac{1}{2}\left|y_{m}^{\prime}(t)\right|^{2}+\frac{1}{2} \hat{M}\left(\left|\nabla y_{m}(t)\right|^{2}\right)+\frac{1}{\gamma+2}\left\|y_{m}(t)\right\|_{\gamma+2, \Gamma_{0}}^{\gamma+2}\right\} \\
& +\left|\nabla y_{m}^{\prime}(t)\right|^{2}=0 .
\end{aligned}
$$

Integrating (3.6) over $(0, \mathrm{t})$ we get

$$
\begin{aligned}
\frac{1}{2}\left|y_{m}^{\prime}(t)\right|^{2} & +\frac{1}{2} \hat{M}\left(\left|\nabla y_{m}(t)\right|^{2}\right)+\frac{1}{\gamma+2}|| y_{m}(t) \|_{\gamma+2, \Gamma_{0}}^{\gamma+2}+\int_{0}^{t}\left|\nabla y_{m}^{\prime}(s)\right|^{2} d s \\
& =\frac{1}{2}\left|y_{1 m}\right|^{2}+\frac{1}{2} \hat{M}\left(\left|\nabla y_{0 m}\right|^{2}\right)+\frac{1}{\gamma+2}|| y_{0 m} \|_{\gamma+2, \Gamma_{0}}^{\gamma+2} .
\end{aligned}
$$

From (3.5) and (3.7) we obtain the first estimate

$$
\left|y_{m}^{\prime}(t)\right|^{2}+\hat{M}\left(\left|\nabla y_{m}(t)\right|^{2}\right)+\left\|y_{m}(t)\right\|_{\gamma+2, \Gamma_{0}}^{\gamma+2}+\int_{0}^{t}\left|\nabla y_{m}^{\prime}(s)\right|^{2} d s \leq L_{1},
$$

where $L_{1}$ is a positive constant independent of $m \in \mathbf{N}$ and $t \geq 0$.

\section{The second estimate.}

Multiplying (3.4) by $g_{j m}(t)$ and summing over $j$ we have

$$
\begin{aligned}
\frac{d}{d t}\left(y_{m}^{\prime}(t), y_{m}(t)\right)+ & M\left(\left|\nabla y_{m}(t)\right|^{2}\right)\left|\nabla y_{m}(t)\right|^{2}+\frac{1}{2} \frac{d}{d t}\left|\nabla y_{m}(t)\right|^{2} \\
& +|| y_{m}(t) \|_{\gamma+2, \Gamma_{0}}^{\gamma+2}=\left|y_{m}^{\prime}(t)\right|^{2} .
\end{aligned}
$$

Integrating (3.10) over $(0, \mathrm{t})$, taking (1.1), (3.5) into account and observing that there exists $C_{0}>0$ such that $|v| \leq C_{0}|\nabla v|, \forall v \in V$, it follows that

$$
\frac{1}{2}\left|\nabla y_{m}(t)\right|^{2}+\int_{0}^{t}\left\|y_{m}(s)\right\|_{\gamma+2, \Gamma_{0}}^{\gamma+2} d s
$$




$$
\leq k_{0}+C_{0}\left|y_{m}^{\prime}(t)\right|\left|\nabla y_{m}(t)\right|+C_{0}^{2} \int_{0}^{t}\left|\nabla y_{m}^{\prime}(s)\right|^{2} d s,
$$

where $k_{0}$ is a positive constant, that is,

$$
\begin{gathered}
\frac{1}{4}\left|\nabla y_{m}(t)\right|^{2}+\int_{0}^{t}|| y_{m}(s) \|_{\gamma+2, \Gamma_{0}}^{\gamma+2} d s \\
\leq k_{0}+\frac{C_{0}^{2}}{2}\left|y_{m}^{\prime}(t)\right|^{2}+C_{0}^{2} \int_{0}^{t}\left|\nabla y_{m}^{\prime}(s)\right|^{2} d s .
\end{gathered}
$$

From (3.11) and considering the first estimate we obtain the second one

$$
\left|\nabla y_{m}(t)\right|^{2}+\int_{0}^{t}\left\|y_{m}(s)\right\|_{\gamma+2, \Gamma_{0}}^{\gamma+2} d s \leq L_{2}
$$

where $L_{2}$ is a positive constant independent of $m \in \mathbf{N}$ and $t \geq 0$.

\section{The third estimate.}

Multiplying (3.4) by $g_{j m}^{\prime \prime}(t)$ and summing over $(0, \mathrm{t})$ we have

$$
\begin{gathered}
\left|y_{m}^{\prime \prime}(t)\right|^{2}+M\left(\left|\nabla y_{m}(t)\right|^{2}\right)\left(\nabla y_{m}(t), \nabla y_{m}^{\prime \prime}(t)\right)+\frac{1}{2} \frac{d}{d t}\left|\nabla y_{m}^{\prime}(t)\right|^{2} \\
+\left(\left|y_{m}(t)\right|^{\gamma} y_{m}(t), y_{m}^{\prime \prime}(t)\right)_{\Gamma_{0}}=0
\end{gathered}
$$

Estimate for $I_{1}:=M\left(\left|\nabla y_{m}(t)\right|^{2}\right)\left(\nabla y_{m}(t), \nabla y_{m}^{\prime \prime}(t)\right)$.

We have

$$
\begin{gathered}
I_{1}=\frac{d}{d t}\left[M\left(\left|\nabla y_{m}(t)\right|^{2}\right)\left(\nabla y_{m}(t), \nabla y_{m}^{\prime}(t)\right)\right] \\
-2 M^{\prime}\left(\left|\nabla y_{m}(t)\right|^{2}\right)\left[\left(\nabla y_{m}(t), \nabla y_{m}^{\prime}(t)\right)\right]^{2}-M\left(\left|\nabla y_{m}(t)\right|^{2}\right)\left|\nabla y_{m}^{\prime}(t)\right|^{2} .
\end{gathered}
$$

Considering that $M \in C^{1}\left(\mathbf{R}_{+}\right)$and the inequality (3.12), we obtain

$$
\begin{gathered}
\left|2 M^{\prime}\left(\left|\nabla y_{m}(t)\right|^{2}\right)\left[\left(\nabla y_{m}(t), \nabla y_{m}^{\prime}(t)\right)\right]^{2}\right| \\
\leq 2\left|M^{\prime}\left(\left|\nabla y_{m}(t)\right|^{2}\right)\right|\left|\nabla y_{m}(t)\right|^{2}\left|\nabla y_{m}^{\prime}(t)\right|^{2} \\
\leq C_{1}\left|\nabla y_{m}^{\prime}(t)\right|^{2}
\end{gathered}
$$


and

$$
\left.\left.\left|M\left(\left|\nabla y_{m}(t)\right|^{2}\right)\right| \nabla y_{m}^{\prime}(t)\right|^{2}\left|\leq C_{2}\right| \nabla y_{m}^{\prime}(t)\right|^{2} .
$$

Then,

$$
\begin{gathered}
\left|y_{m}^{\prime \prime}(t)\right|^{2}+\frac{d}{d t}\left[M\left(\left|\nabla y_{m}(t)\right|^{2}\right)\left(\nabla y_{m}(t), \nabla y_{m}^{\prime}(t)\right)\right] \\
+\frac{1}{2} \frac{d}{d t}\left|\nabla y_{m}^{\prime}(t)\right|^{2}+\frac{d}{d t}\left(\left|y_{m}(t)\right|^{\gamma} y_{m}(t), y_{m}^{\prime}(t)\right)_{\Gamma_{0}} \\
-(\gamma+1)\left(\left|y_{m}(t)\right|^{\gamma} y_{m}^{\prime}(t), y_{m}^{\prime}(t)\right)_{\Gamma_{0}} \leq C_{3}\left|\nabla y_{m}^{\prime}(t)\right|^{2},
\end{gathered}
$$

where $C_{3}$ is the positive constant $C_{1}+C_{2}$.

Estimate for $I_{2}:=(\gamma+1)\left(\left|y_{m}(t)\right|^{\gamma} y_{m}^{\prime}(t), y_{m}^{\prime}(t)\right)_{\Gamma_{0}}$.

Noting that $\frac{\gamma}{2(\gamma+1)}+\frac{1}{2(\gamma+1)}+\frac{1}{2}=1$, the generalized Hölder inequality yields

$$
\left|I_{2}\right| \leq(\gamma+1)\left\|y_{m}(t)\right\|_{2(\gamma+1), \Gamma_{0}}^{\gamma} \|\left. y_{m}^{\prime}(t)\right|_{2(\gamma+1), \Gamma_{0}}\left|y_{m}^{\prime}(t)\right|_{\Gamma_{0}} .
$$

Now, since $V \hookrightarrow L^{2(\gamma+1)}\left(\Gamma_{0}\right)$ then, from the last inequality and considering the second estimate we conclude

$$
\left|I_{3}\right| \leq(\gamma+1)\left|\nabla y_{m}(t)\right|^{\gamma}\left|\nabla y_{m}^{\prime}(t)\right|\left|\nabla y_{m}^{\prime}(t)\right| \leq C_{4}\left|\nabla y_{m}^{\prime}(t)\right|^{2},
$$

where $C_{4}$ is a positive constant.

Combining (3.14) and (3.15) and integrating the obtained result over $(0, \mathrm{t})$ we deduce

$$
\begin{gathered}
\int_{0}^{t}\left|y_{m}^{\prime \prime}(s)\right|^{2} d s+2 M\left(\left|\nabla y_{m}(t)\right|^{2}\right)\left(\nabla y_{m}(t), \nabla y_{m}^{\prime}(t)\right) \\
-2 M\left(\left|\nabla y_{0 m}\right|^{2}\right)\left(\nabla y_{0 m}, \nabla y_{1 m}\right)+\left|\nabla y_{m}^{\prime}(t)\right|^{2}-\left|\nabla y_{1 m}\right|^{2} \\
\leq 2 C_{5} \int_{0}^{t}\left|\nabla y_{m}^{\prime}(s)\right|^{2} d s-2\left(\left|y_{m}(t)\right|^{\gamma} y_{m}(t), y_{m}^{\prime}(t)\right)_{\Gamma_{0}}+2\left(\left|y_{0 m}\right|^{\gamma} y_{0 m}, y_{1 m}\right)_{\Gamma_{0}}
\end{gathered}
$$
where $C_{5}$ is a positive constant.

Estimate for $I_{3}:=-2\left(\left|y_{m}(t)\right|^{\gamma} y_{m}(t), y_{m}^{\prime}(t)\right)_{\Gamma_{0}}$. 
From Cauchy-Schwarz inequality, making use of the inequality $a b \leq$ $\frac{1}{4 \eta} a^{2}+\eta b^{2}(\eta>0$ arbitrary $)$ and considering the second estimate we obtain $k_{1}$ and $C_{6}(\eta)$ positive constants such that

$$
\begin{gathered}
\left|I_{3}\right| \leq 2 \int_{\Gamma_{0}}\left|y_{m}\right|^{\gamma+1}\left|y_{m}^{\prime}\right| d \Gamma \\
\leq 2|| y_{m}(t)||_{2(\gamma+1), \Gamma_{0}}^{\gamma+1}\left|y_{m}^{\prime}(t)\right|_{\Gamma_{0}} \\
\leq k_{1}\left|\nabla y_{m}(t)\right|^{\gamma+1}\left|\nabla y_{m}^{\prime}(t)\right| \\
\leq C_{6}(\eta)+\eta\left|\nabla y_{m}^{\prime}(t)\right|^{2} .
\end{gathered}
$$

Thus, from (3.5), (3.16) and (3.17) there exists a positive constant $C_{7}(\eta)$ such that

$$
\begin{gathered}
\int_{0}^{t}\left|y_{m}^{\prime \prime}(s)\right|^{2} d s+2 M \\
\left(\left|\nabla y_{m}(t)\right|^{2}\right)\left(\nabla y_{m}(t), \nabla y_{m}^{\prime}(t)\right)+(1-\eta)\left|\nabla y_{m}^{\prime}(t)\right|^{2} \\
\leq C_{7}(\eta)+2 C_{5} \int_{0}^{t}\left|\nabla y_{m}^{\prime}(s)\right|^{2} d s .
\end{gathered}
$$

Consequently, from the second estimate and from the last inequality we conclude

$$
\begin{aligned}
& \int_{0}^{t}\left|y_{m}^{\prime \prime}(s)\right|^{2} d s+(1-2 \eta)\left|\nabla y_{m}^{\prime}(t)\right|^{2} \\
& \quad \leq C_{8}(\eta)+2 C_{5} \int_{0}^{t}\left|\nabla y_{m}^{\prime}(s)\right|^{2} d s .
\end{aligned}
$$

where $C_{8}$ is a positive constant.

Finally from (3.18), choosing $\eta$ sufficiently small and making use of Gronwall's lemma we obtain the third estimate

$$
\int_{0}^{t}\left|y_{m}^{\prime \prime}(s)\right|^{2} d s+\left|\nabla y_{m}^{\prime}(t)\right|^{2} \leq L_{3}
$$

where $L_{3}$ is a positive constant independent of $m \in \mathbf{N}$ and $t \in[0, T]$.

From the estimate (3.19) we can extract a subsequence $\left(y_{\mu}\right)$ of $\left(y_{m}\right)$ such that

$$
y_{\mu}^{\prime \prime} \rightarrow y^{\prime \prime} \quad \text { weakly in } L^{2}\left(0, T ; L^{2}(\Omega)\right)
$$

and

$$
y_{\mu}^{\prime} \rightarrow y^{\prime} \text { weakly star in } L^{\infty}(0, T ; V) .
$$




\subsection{Analysis of the nonlinear terms}

From now on we are interested in the convergence of the nonlinear terms.

We define:

$$
\varphi_{m}(t)=\left|\nabla y_{m}(t)\right|^{2} ; \quad t \in[0, T] .
$$

From the second estimate we have

$$
\left|\varphi_{m}(t)\right| \leq C ; \quad \forall m \in \mathbf{N}, \quad \forall t \in[0, T],
$$

where $C$ is a positive constant.

Now, if $t_{1}, t_{2} \in[0, T]$ we obtain

$$
\left|\varphi_{m}\left(t_{1}\right)-\varphi_{m}\left(t_{2}\right)\right| \leq \int_{t_{1}}^{t_{2}}\left|\varphi_{m}^{\prime}(s)\right| d s .
$$

On the other hand, from the second and third estimates we deduce

$$
\varphi_{m}^{\prime}(s)=2\left(\nabla y_{m}(s), \nabla y_{m}^{\prime}(s)\right) \leq C ; \forall m \in \mathbf{N}, \forall s \in[0, T] ;
$$

where $C$ is a positive constant independent of $m$.

Combining (3.24) and (3.25) it follows that

$$
\left|\varphi_{m}\left(t_{1}\right)-\varphi_{m}\left(t_{2}\right)\right| \leq C\left|t_{1}-t_{2}\right| ; \forall m \in \mathbf{N} .
$$

Then, from (3.23) and (3.26) by Arzela-Ascoli's theorem there exists a continuous function $\varphi:[0, T] \rightarrow \mathbf{R}$ such that

$$
\varphi_{m}(t) \rightarrow \varphi(t)
$$

uniformly in $[0, T]$ and, since $M \in C^{1}([0, \infty), \mathbf{R})$, we obtain

$$
M\left(\left|\nabla y_{m}(t)\right|^{2}\right) \rightarrow M(\varphi(t))
$$

uniformly in $[0, T]$.

From the second estimate and noting that $V \hookrightarrow L^{2(\gamma+1)}\left(\Gamma_{0}\right)$ we deduce

$$
\left\{\left|y_{m}\right|^{\gamma} y_{m}\right\} \text { is bounded in } L^{\infty}\left(0, T ; L^{2}\left(\Gamma_{0}\right)\right) .
$$

Also, we note that from the second and third estimates and noting that $\|v\|_{H^{1 / 2}\left(\Gamma_{0}\right)} \leq C|\nabla v|$, for all $v \in V$, we infer

$$
\left\{y_{m}\right\} \text { is bounded in } L^{2}\left(0, T ; H^{1 / 2}\left(\Gamma_{0}\right)\right),
$$




$$
\left\{y_{m}^{\prime}\right\} \text { is bounded in } L^{2}\left(0, T ; L^{2}\left(\Gamma_{0}\right)\right) \text {. }
$$

Since the injection $H^{1 / 2}\left(\Gamma_{0}\right) \hookrightarrow L^{2}\left(\Gamma_{0}\right)$ is continuous and compact, making use of Aubin-Lions theorem, see J. L. Lions [13, Theo. 5.1], there exist $\left\{y_{\mu}\right\}$ a subsequence of $\left\{y_{m}\right\}$ such that

$$
y_{\mu} \rightarrow y \text { strongly in } L^{2}\left(0, T ; L^{2}\left(\Gamma_{0}\right)\right) .
$$

From now on all the eventual subsequences are denoted by the same notation.

Therefore,

$$
y_{\mu} \rightarrow y \text { a.e. in } \Gamma_{0} \times(0, T)
$$

and consequently

$$
\left|y_{\mu}\right|^{\gamma} y_{\mu} \rightarrow|y|^{\gamma} y \text { a.e. in } \Gamma_{0} \times(0, T) .
$$

Combining (3.29) and (3.33) we deduce, thanks to Lions' lemma, see J. L. Lions [13, lemma 1.3],

$$
\left|y_{\mu}\right|^{\gamma} y_{\mu} \rightarrow|y|^{\gamma} y \text { weakly in } L^{2}\left(0, T ; L^{2}\left(\Gamma_{0}\right)\right) \text {. }
$$

Considering the above convergences we can pass to the limit in the approximate system given by (3.4) using standard arguments in order to obtain

$$
\begin{gathered}
y^{\prime \prime}-M(\varphi(t)) \Delta y-\Delta y^{\prime}=0 \quad \text { in } L^{2}\left(0, T ; L^{2}(\Omega)\right), \\
y(0)=y^{0} ; y^{\prime}(0)=y^{1} .
\end{gathered}
$$

Moreover, making use of the generalized Green formula we deduce

$$
M(\varphi(t)) \frac{\partial y}{\partial \nu}+\frac{\partial y_{t}}{\partial \nu}+|y|^{\gamma} y=0 \quad \text { i n } L^{2}\left(0, T ; L^{2}\left(\Gamma_{0}\right)\right) .
$$

Our aim is to show that

$$
\varphi(t)=|\nabla y(t)|^{2} ; t \in[0, T] .
$$

Indeed, multiplying (3.4) by $g_{j m}$, summing over $j$ and integrating over $[0, t]$ we obtain

$$
\int_{0}^{t}\left(y_{m}^{\prime \prime}(s), y_{m}(s)\right) d s+\int_{0}^{t} M\left(\left|\nabla y_{m}(s)\right|^{2}\right)\left|\nabla y_{m}(s)\right|^{2} d s
$$




$$
+\int_{0}^{t}\left(\left|y_{m}(s)\right|^{\gamma} y_{m}(s), y_{m}(s)\right)_{\Gamma_{0}} d s+\int_{0}^{t}\left(\nabla y_{m}^{\prime}(s), \nabla y_{m}(s)\right) d s=0 .
$$

On the other hand, from the first and second estimates and making use of Aubin-Lions theorem one has

$$
y_{m} \rightarrow y \quad \text { strongly in } L^{2}\left(0, T ; L^{2}(\Omega)\right) .
$$

Considering the convergences given by (3.20), (3.27), (3.28), (3.32), (3.34), (3.39) we can pass to the limit in (3.38) to obtain

$$
\begin{gathered}
\lim _{m \rightarrow \infty} \int_{0}^{t}\left(\nabla y_{m}^{\prime}(s), \nabla y_{m}(s)\right) d s \\
=-\int_{0}^{t}\left(y^{\prime \prime}(s), y(s)\right) d s-\int_{0}^{t} M(\varphi(s)) \varphi(s) d s-\int_{0}^{t}\left(|y(s)|^{\gamma} y(s), y(s)\right)_{\Gamma_{0}} d s .
\end{gathered}
$$

Combining (3.35), (3.36) and (3.40) and taking the generalized Green formula into account we deduce

$$
\begin{gathered}
\lim _{m \rightarrow \infty} \int_{0}^{t}\left(\nabla y_{m}^{\prime}(s), \nabla y_{m}(s)\right) d s \\
=\int_{0}^{t} M(\varphi(s))\left[|\nabla y(s)|^{2}-\varphi(s)\right] d s+\int_{0}^{t}\left(\nabla y^{\prime}(s), \nabla y(s)\right) d s .
\end{gathered}
$$

The last equality yields

$$
\begin{gathered}
\lim _{m \rightarrow \infty}\left[\frac{1}{2}\left|\nabla y_{m}(t)\right|^{2}-\frac{1}{2}\left|\nabla y_{0 m}\right|^{2}\right] \\
=\int_{0}^{t} M(\varphi(s))\left[|\nabla y(s)|^{2}-\varphi(s)\right] d s+\frac{1}{2}|\nabla y(t)|^{2}-\frac{1}{2}|\nabla y(0)|^{2} .
\end{gathered}
$$

Then, from (3.41) and considering the convergences in (3.5) and (3.27) we conclude

$$
\begin{gathered}
\left.|\varphi(t)-| \nabla y(t)\right|^{2}\left|\leq 2 \int_{0}^{t} M(\varphi(s))\right||\nabla y(s)|^{2}-\varphi(s) \mid d s \\
\leq\left. C \int_{0}^{t}|| \nabla y(s)\right|^{2}-\varphi(s) \mid d s,
\end{gathered}
$$


where $C$ is a positive constant. Employing Gronwall's lemma, from the last inequality we obtain the desired in (3.37).

We observe that for each fixed $t$ in $[0, \infty)$, we have that $y(t)$ is the weak solution of the Dirichlet-Neumann problem

$$
\mid \begin{aligned}
& -\Delta\left[M\left(|\nabla y|^{2}\right) y+y^{\prime}\right]=-y^{\prime \prime} \text { in } L^{2}\left(0, \infty ; L^{2}(\Omega)\right) \\
& M\left(|\nabla y|^{2}\right) y+y^{\prime}=0 \text { on } \Sigma_{1} \\
& \frac{\partial}{\partial \nu}\left[M\left(|\nabla y|^{2}\right) y+y^{\prime}\right]+|y|^{\gamma} y=0 \quad \text { in } L^{2}\left(0, \infty ; L^{2}\left(\Gamma_{0}\right)\right) .
\end{aligned}
$$

Then, the theory of elliptic problems gives

$$
M\left(|\nabla y|^{2}\right) y+y^{\prime} \in L^{2}\left(0, \infty ; V \cap H^{3 / 2}(\Omega)\right) .
$$

\subsection{Uniqueness}

Let $y$ and $\hat{y}$ be two solutions of problem $(*)$. Denoting $z=y-\hat{y}$, it comes from (3.35) and (3.36) that

$$
\begin{gathered}
\frac{1}{2} \frac{d}{d t}\left|z^{\prime}(t)\right|^{2}+M\left(|\nabla y(t)|^{2}\right)\left(\nabla y(t), \nabla z^{\prime}(t)\right) \\
-M\left(|\nabla \hat{y}(t)|^{2}\right)\left(\nabla \hat{y}(t), \nabla z^{\prime}(t)\right)+\left|\nabla z^{\prime}(t)\right|^{2} \\
+\left(|y(t)|^{\gamma} y(t), z^{\prime}(t)\right)_{\Gamma_{0}}-\left(|\hat{y}(t)|^{\gamma} \hat{y}(t), z^{\prime}(t)\right)_{\Gamma_{0}}=0 .
\end{gathered}
$$

Summing and subtracting the term $M\left(|\nabla y(t)|^{2}\right)\left(\nabla \hat{y}(t), \nabla z^{\prime}(t)\right)$ in (3.43) we obtain

$$
\begin{aligned}
& \frac{1}{2} \frac{d}{d t}\left|z^{\prime}(t)\right|^{2}+M\left(|\nabla y(t)|^{2}\right)\left(\nabla z(t), \nabla z^{\prime}(t)\right) \\
&+\left|\nabla z^{\prime}(t)\right|^{2}=\left(M\left(|\nabla \hat{y}(t)|^{2}\right)-M\left(|\nabla y(t)|^{2}\right)\right)\left(\nabla \hat{y}(t), \nabla z^{\prime}(t)\right) \\
&+\left(|\hat{y}(t)|^{\gamma} \hat{y}(t)-|y(t)|^{\gamma} y(t), z^{\prime}(t)\right)_{\Gamma_{0}} .
\end{aligned}
$$

On the other hand, since $M$ is $C^{1}$ we get

$$
\left|M\left(|\nabla \hat{y}(t)|^{2}\right)-M\left(|\nabla y(t)|^{2}\right)\right|
$$




$$
\begin{gathered}
\leq\left. k_{1}|| \nabla y(t)\right|^{2}-|\nabla \hat{y}(t)|^{2} \mid \\
\leq k_{1}[|\nabla y(t)|+|\nabla \hat{y}(t)|]|\nabla y(t)-\nabla \hat{y}(t)| \\
\leq k_{2}|\nabla z(t)|
\end{gathered}
$$

where $k_{1}$ and $k_{2}$ are positive constants.

Combining (3.44) and (3.45) and using analogous considerations like those ones used in the third estimate (see estimate for $I_{3}$ ) it follows that

$$
\begin{gathered}
\frac{1}{2} \frac{d}{d t}\left|z^{\prime}(t)\right|^{2}+\left|\nabla z^{\prime}(t)\right|^{2} \\
\leq C_{1}|\nabla z(t)|\left|\nabla z^{\prime}(t)\right|+C_{2}(\gamma) \int_{\Gamma_{0}}\left(|\hat{y}|^{\gamma}+|y|^{\gamma}\right)|z|\left|z^{\prime}\right| d \Gamma \\
\leq C_{3}|\nabla z(t)||\nabla z \prime(t)| \\
\leq \frac{C_{3}^{2}}{2}|\nabla z(t)|^{2}+\frac{1}{2}\left|\nabla z^{\prime}(t)\right|^{2} .
\end{gathered}
$$

Integrating $(3.46)$ over $(0, \mathrm{t})$ we obtain

$$
\begin{gathered}
\frac{1}{2}\left|z^{\prime}(t)\right|^{2}+\frac{1}{2} \int_{0}^{t}\left|\nabla z^{\prime}(s)\right|^{2} d s \\
\leq \frac{C_{3}^{2}}{2} \int_{0}^{t}|\nabla z(s)|^{2} d s .
\end{gathered}
$$

Since

$$
|\nabla z(t)|^{2} \leq T \int_{0}^{t}\left|\nabla z^{\prime}(s)\right|^{2} d s
$$

combining (3.47) and (3.48) and applying Gronwall's lemma we obtain $|\nabla z(t)|=\left|z^{\prime}(t)\right|=0$. This concludes the uniqueness of strong solutions.

\subsection{Solvability of weak solutions}

We have just proved the existence of solutions to problem $(*)$ when $\left\{y^{0}, y^{1}\right\} \in V^{2}$. However, when $\left\{y^{0}, y^{1}\right\} \in V \times L^{2}(\Omega)$, by density arguments and using analogous considerations like those ones used in the first and second estimates and in the uniqueness, we can find a sequence $\left\{y_{\mu}\right\}$ of solutions to problem $(*)$ and a function $y: \Omega \times(0, T) \rightarrow \mathbf{R}$ such that

$$
y_{\mu} \in L^{\infty}(0, \infty ; V), \quad y_{\mu}^{\prime} \in L^{\infty}(0, \infty ; V), \quad y_{\mu}^{\prime \prime} \in L^{2}\left(0, \infty ; L^{2}(\Omega)\right),
$$




$$
\begin{gathered}
y_{\mu} \rightarrow y \text { strongly in } C^{0}([0, T] ; V) \\
y_{\mu}^{\prime} \rightarrow y^{\prime} \text { strongly in } C^{0}\left([0, T] ; L^{2}(\Omega)\right) \\
y_{\mu}^{\prime} \rightarrow y^{\prime} \text { strongly in } L^{2}(0, T ; V) \\
y_{\mu}^{\prime} \rightarrow y^{\prime} \text { weakly star in } L^{\infty}(0, T ; V)
\end{gathered}
$$

and

$$
y_{\mu}^{\prime \prime}-M\left(\left|\nabla y_{\mu}\right|^{2}\right) \Delta y_{\mu}-\Delta y_{\mu}^{\prime}=0 \quad \text { in } \quad L^{2}\left(0, T ; L^{2}(\Omega) .\right.
$$

Consider $\theta \in D(0, T)$ and $\phi \in D(\Omega)$. Then,

$$
\begin{gathered}
-\int_{0}^{T}\left(y_{\mu}^{\prime}(t), \phi\right) \theta^{\prime}(t) d t+\int_{0}^{T} M\left(\left|\nabla y_{\mu}(t)\right|^{2}\right)\left(\nabla y_{\mu}(t), \nabla \phi\right) \theta(t) d t \\
+\int_{0}^{T}\left(\nabla y_{\mu}^{\prime}(t), \nabla \phi\right) \theta(t) d t=0 .
\end{gathered}
$$

The above convergences are sufficient to pass to the limit in the above equality in order to obtain

$$
\begin{gathered}
-\int_{0}^{T}\left(y^{\prime}(t), \phi\right) \theta^{\prime}(t) d t+\int_{0}^{T} M\left(|\nabla y(t)|^{2}\right)(\nabla y(t), \nabla \phi) \theta(t) d t \\
+\int_{0}^{T}\left(\nabla y^{\prime}(t), \nabla \phi\right) \theta(t) d t=0,
\end{gathered}
$$

for all $\theta \in D(0, T)$ and $\phi \in D(\Omega)$.

Consequently,

$$
\left\langle-\left\langle y^{\prime}, \theta^{\prime}\right\rangle, \phi\right\rangle-\left\langle\left\langle M\left(|\nabla y|^{2}\right) \Delta y, \theta\right\rangle, \phi\right\rangle-\left\langle\left\langle\Delta y^{\prime}, \theta\right\rangle, \phi\right\rangle=0,
$$

for all $\theta \in D(0, T), \phi \in D(\Omega)$; where $\langle\cdot, \theta\rangle$ and $\langle\cdot, \phi\rangle$ represent the duality $D^{\prime}(0, T) \times D(0, T)$ and $D^{\prime}(\Omega) \times D(\Omega)$, respectively.

Then,

$$
\left\langle y^{\prime \prime}, \theta \phi\right\rangle-\left\langle M\left(|\nabla y|^{2}\right) \Delta y, \theta \phi\right\rangle-\left\langle\Delta y^{\prime}, \theta \phi\right\rangle=0,
$$

for all $\theta \in D(0, T), \phi \in D(\Omega)$; that is, 


$$
y^{\prime \prime}-M\left(|\nabla y|^{2}\right) \Delta y-\Delta y^{\prime}=0 \quad \text { in } \quad D^{\prime}(\Omega \times] 0, T[),
$$

since $\{\theta \phi ; \theta \in D(0, T), \phi \in D(\Omega)\}$ is dense in $D(\Omega \times] 0, T[)$.

However, $\Delta y, \Delta y^{\prime} \in L^{2}\left(0, T: V^{\prime}\right)$, which implies that

$$
y^{\prime \prime} \in L^{2}\left(0, T ; V^{\prime}\right),
$$

provided that $y^{\prime \prime}=M\left(|\nabla y|^{2}\right) \Delta y+\Delta y^{\prime}$ in $D^{\prime}(\Omega \times] 0, T[)$. Then, we obtain a weak solution $y$ to $(*)$ in the class

$$
\begin{aligned}
& y \in C^{0}([0, \infty) ; V) ; y^{\prime} \in C^{0}([0, \infty) ; \\
& \left.L^{2}(\Omega)\right) \cap L^{2}(0, \infty ; V) ; y^{\prime \prime} \in L^{2}\left(0, \infty ; V^{\prime}\right)
\end{aligned}
$$

verifying

$$
y^{\prime \prime}-M\left(|\nabla y|^{2}\right) \Delta y-\Delta y^{\prime}=0 \quad \text { in } \quad L^{2}\left(0, \infty ; V^{\prime}\right) .
$$

\subsection{Characterization of the boundary condition}

Let us consider the elliptic problem

$$
\mid \begin{aligned}
& -\Delta p=y^{\prime} \text { in } \Omega \\
& p=0 \text { on } \Gamma_{1} \\
& \frac{\partial p}{\partial \nu}=\int_{0}^{t}|y(s)|^{\gamma} y(s) d s \text { on } \Gamma_{0}
\end{aligned}
$$

where $y$ is the weak solution of $(*)$ verifying (3.53). Taking the regularity of $\Gamma$ into account, we have

$$
p \in L^{2}(0, \infty ; \mathcal{H})
$$

where

$$
\mathcal{H}=\left\{u \in V ; \Delta u \in L^{2}(\Omega)\right\} .
$$

Next, we are going to prove that

$$
M\left(|\nabla y|^{2}\right) y+y^{\prime}=-p^{\prime} \quad \text { in } \quad H^{-1}(0, \infty ; \mathcal{H}) .
$$

Indeed, from (3.53) we can write

$$
-\Delta\left[M\left(|\nabla y|^{2}\right) y+y^{\prime}\right]=-y^{\prime \prime} \text { in } L_{l o c}^{2}\left(0, \infty ; V^{\prime}\right)
$$


and from (3.56) we obtain

$$
-\Delta\left[M\left(|\nabla y|^{2}\right) y+y^{\prime}\right]=\Delta p^{\prime} \text { in } D_{l o c}^{\prime}\left(0, \infty ; V^{\prime}\right) .
$$

Therefore

$$
\left\langle-\Delta\left[M\left(|\nabla y|^{2}\right) y+y^{\prime}\right], \theta\right\rangle=\left\langle-\Delta p, \theta^{\prime}\right\rangle \text { in } V^{\prime} \text {, for all } \theta \in D(0, \infty) \text {. }
$$

Hence,

$$
\int_{0}^{T}(-\Delta)\left(M\left(|\nabla y|^{2}\right) y+y^{\prime}\right)(t) \theta(t) d t=\int_{0}^{T}(-\Delta) p(t) \theta^{\prime}(t) d t \quad \text { in } \quad V^{\prime}
$$

and consequently

$$
\Delta \int_{0}^{T}\left(M\left(|\nabla y|^{2}\right) y+y^{\prime}\right) \theta(t) d t=\Delta \int_{0}^{T} p(t) \theta^{\prime}(t) d t \quad \text { in } \quad V^{\prime} .
$$

In order to prove (3.56) it remains to prove that the operator $-\Delta$ : $V \rightarrow V^{\prime}$ is an injective map. In fact, if this afirmative is true we conclude from (3.57) that

$$
\int_{0}^{T}\left(M\left(|\nabla y|^{2}\right) y+y^{\prime}\right) \theta(t) d t=\int_{0}^{T} p(t) \theta(t) d t \quad \text { in } \quad V
$$

for all $\theta \in D(0, \infty)$, and, consequently, (3.56) follows from the above inequality and from (3.55). To prove the desired result, let us define the following

$$
\begin{gathered}
a(u, v)=\int \nabla u \cdot \nabla v d x ; \quad \text { for } \quad \text { all } \quad u, v \in V \\
L(v)=\int_{\Gamma_{0}} g v d \Gamma+\int_{\Omega} f v d x ; \quad \text { for } \quad \text { all } \quad v \in V,
\end{gathered}
$$

where $g \in L^{2}\left(\Gamma_{0}\right)$ and $f \in L^{2}(\Omega)$. From the above definitions we conclude from Lax-Milgran's lemma that there exists a unique $u \in V$ such that

$$
a(u, v)=L(v), \quad \text { for } \quad \text { all } \quad v \in V .
$$

In other words, there exists a unique $u \in V$ verifying 


$$
\mid \begin{aligned}
& -\Delta u=f \quad \text { in } \Omega \\
& u=0 \text { on } \Gamma_{1} \\
& \frac{\partial u}{\partial \nu}=g \text { on } \Gamma_{0} .
\end{aligned}
$$

Then, given $\{f, g\} \in L^{2}(\Omega) \times L^{2}\left(\Gamma_{0}\right)$, the solution of the above elliptic problem is uniquely determined, which proves that the operator $-\Delta: V \rightarrow V^{\prime}$ is an injective map.

In what follows we are going to consider the dual trace operators

$$
\begin{gathered}
\hat{\gamma}_{0}: H_{l o c}^{-1}(0, \infty ; V) \rightarrow H_{l o c}^{-1}\left(0, \infty ; L^{2}\left(\Gamma_{0}\right)\right), \\
\hat{\gamma}_{1}: H_{l o c}^{-1}(0, \infty ; \mathcal{H}) \rightarrow H_{l o c}^{-1}\left(0, \infty ; H^{-1 / 2}\left(\Gamma_{0}\right)\right),
\end{gathered}
$$

whose constructions and properties can be found in Milla Miranda [15].

From (3.56) we obtain

$$
\left(\left.\hat{\gamma}_{1}\right|_{\Sigma_{0}}\right)\left(M\left(|\nabla y|^{2}\right) y+y^{\prime}\right)=-\left(\left.\hat{\gamma}_{1}\right|_{\Sigma_{0}}\right)\left(p^{\prime}\right)=-\left(\bar{\gamma}_{1}(p)\right)^{\prime},
$$

where

$$
\bar{\gamma}_{1}: L_{l o c}^{2}(0, \infty ; \mathcal{H}) \rightarrow L_{l o c}^{2}\left(0, \infty ; H^{-1 / 2}\left(\Gamma_{0}\right)\right)
$$

is the trace operator given by

$$
\left(\bar{\gamma}_{1} u\right)(t)=\gamma_{1}(u(t))
$$

and $\gamma_{1}: \mathcal{H} \rightarrow H^{-1 / 2}\left(\Gamma_{0}\right)$ is the usual one related to the normal derivative.

Thus, from (3.54) and (3.60) we conclude

$$
\begin{gathered}
\left(\left.\hat{\gamma}_{1}\right|_{\Sigma_{0}}\right)\left(M\left(|\nabla y|^{2}\right) y+y^{\prime}\right)=-\left(\bar{\gamma}_{0}\left(\int_{0}^{t}|y(s)|^{\gamma} y(s) d s\right)\right)^{\prime} \\
=-\left(\hat{\gamma}_{0} \mid \Sigma_{0}\right)\left(|y|^{\gamma} y\right)
\end{gathered}
$$

where, analogously,

$$
\bar{\gamma}_{0}: L_{l o c}^{2}(0, \infty ; V) \rightarrow L_{l o c}^{2}\left(0, \infty ; L^{2}\left(\Gamma_{0}\right)\right)
$$

is the trace operator defined by

$$
\left(\bar{\gamma}_{0} u\right)(t)=\gamma_{0}(u(t)) .
$$


Hence, from (3.61) we can give meaning to the boundary condition. More precisely we have

$$
\frac{\partial}{\partial \nu}\left(M\left(|\nabla y|^{2}\right) y+y^{\prime}\right)+|y|^{\gamma} y=0 \quad \text { in } \quad H_{l o c}^{-1}\left(0, \infty ; L^{2}\left(\Gamma_{0}\right)\right)
$$

Since $|y|^{\gamma} y \in L^{2}\left(0, \infty ; L^{2}\left(\Gamma_{0}\right)\right)$ we have the boundary characterization valid a.e. on $\Sigma_{0}$.

\subsection{Uniqueness}

According to (3.53) we have

$$
y^{\prime \prime}-M\left(|\nabla y|^{2}\right) \Delta y-\Delta y^{\prime}=0 \quad \text { in } \quad L^{2}\left(0, T ; V^{\prime}\right) .
$$

Let $y$ and $\hat{y}$ be two weak solutions of problem (*). Defining $z=y-\hat{y}$ we have that $z \in H^{1}(0, T, V)$ and $\mathrm{z}$ satisfies

$$
z^{\prime \prime}-\Delta\left(M|\nabla y|^{2} y-y^{\prime}\right)+\Delta\left(M|\nabla \hat{y}|^{2} \hat{y}-\hat{y}\right)=0 \quad \text { in } \quad L^{2}\left(0, T ; V^{\prime}\right) .
$$

Then, we are able to compose the above equation with $z^{\prime}$, and following step by step the arguments done in the case of strong solutions, we conclude that $|\nabla z(t)|=\left|z^{\prime}(t)\right|=0$; which proves the uniqueness.

\section{Asymptotic behaviour}

In this section we are going to obtain the algebraic decay for strong solutions of $(*)$. Using density arguments we obtain the same result for weak solutions.

The derivative of the energy defined in (1.3) is given by

$$
E^{\prime}(t)=-\left|\nabla y^{\prime}(t)\right|^{2}
$$

Let us consider the perturbed energy

$$
E_{\varepsilon}(t)=E(t)+\varepsilon \psi(t),
$$

where

$$
\psi(t)=\left(y^{\prime}(t), y(t)\right)+\frac{1}{2}|\nabla y(t)|^{2} .
$$


Taking the derivative of (4.2) with respect to $t$, replacing

$$
\begin{aligned}
& \left(y^{\prime \prime}(t), y(t)\right)=-M\left(|\nabla y(t)|^{2}\right)|\nabla y(t)|^{2}-\left(\nabla y^{\prime}(t), \nabla y(t)\right)- \\
& \left(|y(t)|^{\gamma} y(t), y(t)\right)_{\Gamma_{0}}
\end{aligned}
$$

in the obtained result and considering (4.1), it follows that

$$
\begin{gathered}
E_{\varepsilon}^{\prime}(t)=E^{\prime}(t)+\varepsilon \psi^{\prime}(t) \\
=-\left|\nabla y^{\prime}(t)\right|^{2}-\varepsilon M\left(|\nabla y(t)|^{2}\right)|\nabla y(t)|^{2}-\varepsilon|| y(t) \|_{\gamma+2, \Gamma_{0}}^{\gamma+2}+\varepsilon\left|y^{\prime}(t)\right|^{2} .
\end{gathered}
$$

Let $\mu$ be a positive constant such that

$$
|v|^{2} \leq \mu|\nabla v|^{2}, \quad \forall v \in V .
$$

From (4.4) and (4.5) we obtain

$$
E_{\varepsilon}^{\prime}(t) \leq\left(-1+\frac{3}{2} \varepsilon \mu\right)\left|\nabla y^{\prime}(t)\right|^{2}-\varepsilon M\left(|\nabla y(t)|^{2}\right)|\nabla y(t)|^{2}-\varepsilon\|y(t) \mid\|_{\gamma+2, \Gamma_{0}}^{\gamma+2} .
$$

Besides, taking into account that $\mathrm{M}$ is a non decreasing function we have

$$
\hat{M}\left(|\nabla y(t)|^{2}\right) \leq M\left(|\nabla y(t)|^{2}\right)|\nabla y(t)|^{2}
$$

and therefore (4.6) yields

$$
E_{\varepsilon}^{\prime}(t) \leq-\left(1-\frac{3}{2} \varepsilon \mu\right)\left|\nabla y^{\prime}(t)\right|^{2}-\varepsilon E(t)
$$

Considering $\varepsilon \in\left(0, \varepsilon_{1}\right]$, where $\varepsilon_{1}=\frac{2}{3 \mu}$, we deduce

$$
E_{\varepsilon}^{\prime}(t) \leq-\varepsilon E(t)
$$

and, consequently, integrating the last inequality over $[0, t]$ it follows that

$$
\varepsilon \int_{0}^{t} E(s) d s \leq E_{\varepsilon}(0)-E_{\varepsilon}(t) \leq L,
$$

where $L$ is a positive constant which depends only on the initial data $\left\{y^{0}, y^{1}\right\}$. 
Thus, from (4.9) we have

$$
\int_{0}^{t} E(s) d s \leq \frac{L}{\varepsilon},
$$

which implies that

$$
E \in L^{1}(0, \infty) .
$$

On the other hand, since $E^{\prime}(t) \leq 0$, we have

$$
(1+t) E^{\prime}(t) \leq 0, \quad \forall t \geq 0,
$$

then

$$
\frac{d}{d t}[(1+t) E(t)] \leq E(t) .
$$

Integrating (4.11) and taking (4.10) into account we obtain

$$
E(t) \leq\left[E(0)+\int_{0}^{\infty} E(s) d s\right](1+t)^{-1}
$$

which concludes the desired in (2.4).

From now on we assume that hypothesis (2.5) hold. Then, we conclude the following result

Lemma 4.1. We have

$$
\lim _{t \rightarrow \infty}|\nabla y(t)|^{2}=0
$$

Proof. Assume that (4.13) does not hold. Then, there exists $\varepsilon_{0}>0$ such that for every $n \in \mathbf{N}$ there exists $t_{n}$, with $t_{n}>n$ and

$$
\left|\nabla y\left(t_{n}\right)\right|^{2} \geq \varepsilon_{0} .
$$

From the last inequality and since $\hat{M}$ is a non decreasing function we have

$$
\hat{M}\left(\left|\nabla y\left(t_{n}\right)\right|^{2}\right) \geq \hat{M}\left(\varepsilon_{0}\right) \geq 0 ; \quad t_{n}>n .
$$

Consequently from (4.12) and (4.14) we obtain

$$
\hat{M}\left(\varepsilon_{0}\right)=0 .
$$


On the other hand, from assumption (2.5) we have

$$
\liminf _{s \rightarrow 0^{+}} \frac{M(s)}{s^{\alpha}}=l>0,
$$

which implies the existence of a real number $\delta_{1}>0$ such that

$$
M(s)>\frac{l}{2} s^{\alpha} ; \quad \forall s \in\left(0, \delta_{1}\right)
$$

and

$$
M(s)>0, \quad \forall s \in\left(0, \delta_{1}\right)
$$

Defining

$$
\delta=\min \left\{\delta_{1}, \varepsilon_{0}\right\}
$$

we have from (4.15) that

$$
0 \leq \int_{0}^{\delta} M(\lambda) d \lambda \leq \int_{0}^{\varepsilon_{0}} M(\lambda) d \lambda=\hat{M}\left(\varepsilon_{0}\right)=0,
$$

that is,

$$
\int_{0}^{\delta} M(\lambda) d \lambda=0 .
$$

But (4.18) it is a contradiction with (4.17). This proves the lemma.

Next, we are going to prove that there exist $C_{1}$ and $N$ positive constant such that

$$
|\nabla y(t)|^{2(\alpha+1)} \leq C_{1} \hat{M}\left(|\nabla y(t)|^{2}\right) ; \quad t>N .
$$

Indeed, from (4.13) there exists $N>0$ such that

$$
|\nabla y(t)|^{2} \leq \delta_{1} ; \quad t>N .
$$

On the other hand, integrating $(4.16)$ over $(0, t)$ it follows that

$$
\frac{l}{2} t^{\alpha+1} \leq \hat{M}(t), \quad \forall t \in\left(0, \delta_{1}\right) .
$$

Combining (4.20) and (4.21) we conclude the desired in (4.19). 
From (4.2),(4.3) and (4.5) we can write

$$
\begin{gathered}
E_{\varepsilon}(t) \geq\left(\frac{1}{2}-\mu \varepsilon\right)\left|y^{\prime}(t)\right|^{2}+\frac{\varepsilon}{4}|\nabla y(t)|^{2}+\frac{1}{2} \hat{M}\left(|\nabla y(t)|^{2}\right) \\
+\frac{\varepsilon}{\gamma+2}\|y(t)\|_{\gamma+2, \Gamma_{0}}^{\gamma+2} .
\end{gathered}
$$

Then,

$$
E_{\varepsilon}(t) \geq 0, \quad \forall \varepsilon \in(0,1 / 2 \mu] .
$$

From (4.2), (4.3), (4.5), (4.12) and (4.19) we have for all $\varepsilon \leq$ $\left\{\varepsilon_{1}, \frac{1}{2 \mu}, 1\right\}$ and $t>N$ that

$$
\begin{gathered}
E_{\varepsilon}(t) \leq E(t)+\frac{\varepsilon}{2}\left|y^{\prime}(t)\right|^{2}+\frac{C_{1} \varepsilon}{2}(\mu+1)\left[\hat{M}\left(|\nabla y(t)|^{2}\right)\right]^{\frac{1}{\alpha+1}} \\
\leq E(t)+\varepsilon E(t)+C_{1} \varepsilon(\mu+1)[E(t)]^{\frac{1}{\alpha+1}} \\
\leq C_{2}[E(t)]^{\frac{1}{\alpha+1}}
\end{gathered}
$$

and therefore

$$
\left[E_{\varepsilon}(t)\right]^{\alpha+1} \leq C E(t), \quad t>N .
$$

Combining (4.8) and (4.24), it follows that

$$
E_{\varepsilon}^{\prime}(t)+\frac{\varepsilon}{C} E_{\varepsilon}^{\alpha+1}(t) \leq 0, \quad t>N .
$$

Taking (4.23) into account and considering in (4.25) the change of variables

$$
z_{\varepsilon}(t)=E_{\varepsilon}^{-\alpha}(t),
$$

for a fixed and sufficiently small $\varepsilon$, we obtain

$$
z_{\varepsilon}^{\prime}(t) \geq \frac{\varepsilon \alpha}{C_{2}} .
$$

Integrating the last inequality over $[0, \mathrm{t}]$ we get

$$
z_{\varepsilon}(t) \geq z_{\varepsilon}(0)+\frac{\varepsilon \alpha}{C_{2}} t
$$

and, consequently,

$$
E_{\varepsilon}^{-\alpha}(t) \geq C_{3}(1+t) .
$$


From the last inequality we conclude that there exists a positive constant $C$, which depends on the initial data, such that

$$
E_{\varepsilon}(t) \leq C(1+t)^{-\frac{1}{\alpha}}, \quad t>N .
$$

This result combined with (4.22) proves (2.6). Finally, since $M(s)=$ $O\left(s^{\alpha}\right), s \rightarrow 0$ we deduce (2.7) from (2.6).

Finally, if we have $M(\lambda) \geq \lambda_{0}>0$, then from (4.2) and (4.3), there exists $\theta_{1}>0$ such that

$$
\left|E_{\varepsilon}(t)-E(t)\right| \leq \varepsilon \theta_{1} E(t) .
$$

Then, combining (4.8) and (4.28) we obtain the exponential decay mentioned in (2.9). The proof is now completed.

\section{Further remarks}

(1) Let $-\Delta$ the operator defined by the triple $\left\{V, L^{2}(\Omega), a(u, v)\right\}$ where

$$
a(u, v)=\int_{\Omega} \nabla u \cdot \nabla v d x, u, v \in V
$$

and

$$
D(-\Delta)=\left\{u \in V \cap H^{2}(\Omega) ; \frac{\partial u}{\partial \nu}=0 \text { on } \Gamma_{0}\right\} .
$$

We recall that the that Spectral Theorem for self-adjoint operators guarantees the existence of a complete orthonormal system $\left(\omega_{\nu}\right)$ of $L^{2}(\Omega)$ given by the eigenfunctions of $-\Delta$. If $\left(\lambda_{\nu}\right)$ are the eigenvalues of $-\Delta$, then $\lambda_{\nu} \rightarrow+\infty$ as $\nu \rightarrow+\infty$. Now, since $-\Delta$ is postive, given $\alpha>0$ one has

$$
D\left[(-\Delta)^{\alpha}\right]=\left\{u \in L^{2}(\Omega) ; \sum_{\nu=1}^{\infty} \lambda_{\nu}^{2 \alpha}\left|\left(u, \omega_{\nu}\right)\right|^{2}<\infty\right\}
$$

and

$$
(-\Delta)^{\alpha} u=\sum_{\nu==1}^{\infty} \lambda_{\nu}^{\alpha}\left(u, \omega_{\nu}\right) \omega_{\nu}, \quad \text { for all } \quad u \in D\left[(-\Delta)^{\alpha}\right] .
$$

In $D\left[(-\Delta)^{\alpha}\right]$ we consider the topology given by

$$
\|u\|_{D\left[-(\Delta)^{\alpha}\right]}=\left|(-\Delta)^{\alpha} u\right|_{L^{2}(\Omega)} .
$$


We observe that such operators are self-adjoints, that is,

$$
\left((-\Delta)^{\alpha} u, v\right)=\left(u,(-\Delta)^{\alpha} v\right) ; \quad \text { for all } \quad u, v \in D\left[(-\Delta)^{\alpha}\right] \text {, }
$$

and, moreover, $D\left[(-\Delta)^{1 / 2}\right]=V$.

Choosing smooth initial data, considering a basis in $D\left[(-\Delta)^{\alpha}\right]$, for $\alpha \geq 1$, and noting that the injection $D\left[(-\Delta)^{\frac{\alpha}{2}}\right] \hookrightarrow D\left[(-\Delta)^{\frac{1}{2}}\right]$ is continuous we can repeat all the considerations used in the above estimates in order to extend our results for a general class of damped problems

$$
\left\{\begin{array}{l}
y_{t t}-M\left(\int_{\Omega}|\nabla y|^{2} d x\right) \Delta y+(-\Delta)^{\alpha} y_{t}=0 \text { in } Q \\
y=0 \text { on } \Sigma_{1} \\
M\left(\int_{\Omega}|\nabla y|^{2} d x\right) \frac{\partial y}{\partial \nu}+\frac{\partial}{\partial \nu}\left[(-\Delta)^{\alpha-1} y_{t}\right]+|y|^{\gamma} y=0 \text { on } \Sigma_{0} \\
y(0)=y^{0} ; \quad y_{t}(0)=y^{1} \quad \text { in } \Omega .
\end{array}\right.
$$

(2) We observe that we could avoid the characterization of the boundary condition if we use Semigroup theory. The linear part of the system generates an analytic semigroup and the nonlinear terms could be treated as perturbations, and it is enough to show solvability for small times. In spite of all these facilities we need the a priori estimates to show global solvability.

\section{Acknowledgement.}

The authors are grateful to the referee for the constructive comments and useful suggestions.

\section{References}

[1] A. Arosio - S. Spagnolo, Global solutions of the Cauchy problem for a nonlinear hyperbolic equation, Nonlinear Differential Equations and Their Applications (edited by H. Brezis and J. L. Lions), Collège de France Seminar,6, Pitman, London (1984).

[2] M. M. Cavalcanti, V. N. Domingos Cavalcanti \& J. A. Soriano, Existence and Boundary Stabilization of a Nonlinear Hyperbolic Equation with TimeDependent Coefficients, EJDE, 1998(8), (1998), 1-21. 
[3] M. M. Cavalcanti, V. N. Domingos Cavalcanti, J. S. Prates Filho \& J. A. Soriano, Existence and Exponential Decay for a Kirchhoff-Carrier Model with Viscosity, J. Math. Anal. Appl., 226(1998), 20-40.

[4] G. Chen, A Note on the Boundary Stabilization of the Wave Equation, Siam J. Control and Optimization, 19(1), (1981), 106-113.

[5] Y. Ebihara, M. Milla Miranda \& L. A. Medeiros, Local solutions for a nonlinear degenerated hyperbolic equation, Nonlinear Anal. Theory, Meth. and Appl., 10(1), (1986), 27-40.

[6] A. Friedman, Variational Principles and Free-Boundary Problems, John Wiley \& Sons, (1982).

[7] V. Komornik \& E. Zuazua, A Direct Method for Boundary Stabilization of the Wave Equation, J. Math. Pures et Appl. 69, (1990), 33-54.

[8] J. E. Lagnese, Decay of Solution of the Wave Equations in a Bounded Region with Boundary Dissipation, Journal of Differential Equation, 50, (1983), 163-182.

[9] J. E. Lagnese, Note on Boundary Stabilization of the Wave Equations, Siam J. Control and Optimization, 26, N.5, (1988), 1250-1257.

[10] I. Lasiecka \& D. Tataru, Uniform Boundary Stabilization of Semilinear Wave Equations with Nonlinear Boundary Damping, Diff. and Integral Equations, 6(2), (1993), 507-533.

[11] I. Lasiecka \& J. Ong, Global Solvability and Uniform Decays of Solutions to Quasilinear Equation with Nonlinear Boundary Conditions, Communications in PDE's, 24, (1999), 2069-2109.

[12] J. L. Lions, On some questions in boundary value problems of Mathematical Physics, in Contemporary Developments in Continuum Mechanics and Partial Differential Equations ( edited by G. M. de La Penha and L. A. Medeiros), North-Holland, Amsterdam,(1978).

[13] J. L. Lions - Quelques Méthodes de Résolution des Problemes aux Limites Non-Lineares, Dunod, paris, 1969.

[14] L. A. Medeiros \& M. Milla Miranda, On a nonlinear wave equation with damping, Revista Matemática de la Universidad Complutense de Madrid, 3 (2), (1990), 613-627.

[15] M. Milla Miranda, Traço para o dual dos Espacos de Şobolev, Bol. Soc. Paran. Mat. (2 $2^{a}$ série), 11(2) , (1990), 131-157.

[16] M. Milla Miranda \& P. San Gil Jutuca, Existence and Boundary Stabilization of Solutions for the Kirchhoff Equation, Communications in PDE's, 24, (1999), 1759-1801. 
[17] G. P. Menzala, On classical solutions of a quasi-linear hyperbolic equation, Nonlinear Anal. T. M. A. 3, (1979), 613-627.

[18] J. E. Muñoz Rivera, Smooth effect and decay on a class of non linear evolution equation, Ann. de la Fac. des Sci. de Toulouse, 1(2), (1992), 237-260.

[19] K. Nishihara \& Y. Yamada, On global solutions of some degenerate quasilinear hyperbolic equations with dissipative terms, Funkcial. Ekvac. 33, (1990), 151-159.

[20] J. Quinn \& D. L. Russel, Asymptotic Stability and Energy Decay for Solutions of Hyperbolic Equations, with Boundary Damping, Proceedings of the Royal Society of Edinburg, 77A, (1977), 97-127.

[21] P. H. Rivera, On local strong solutions of a nonlinear partial differential equation, Applicable Analysis, 10, (1980), 93-104.

[22] M. Tusnack, Boundary stabilization for the stretched string equation, Diff. and Int. Equations, 6(4), (1993), 925-935.

[23] C. F. Vasconcellos \& L. M. Teixeira, Strong solutions and exponential decay for a nonlinear hyperbolic equation, Applicable Analysis, 51, (1993), 155173 .

[24] Y. Yamada, On the decay of solutions for some nonlinear evolution equations of second order, Nagoya Math. Journal 73, (1970), 69-98.

[25] Y. Yamada, Some nonlinear degenerate wave equations, NonLinear Analysis, The. Meth. and App., 1 (10), (1987), 1155-1168.

[26] E. Zuazua, Stability and decay for a class of nonlinear hyperbolic problems, Asymptotic Analysis,1(1988), 161-185.

Departamento de Matemática Universidade Estadual de Maringá 87020-900 Maringá-PR, Brasil
Recibido: 26 de Octubre de 1999

Revisado: 3 de Agosto de 2000 